\title{
Inverse eigenvalue problem for a simple star graph
}

\author{
William Rundell ${ }^{1,2}$ and Paul Sacks ${ }^{2}$
}

\begin{abstract}
A Schrödinger operator and associated spectra may be defined for a graph by identifying edges with intervals of $\mathbb{R}$, on which coefficient functions are defined, imposing appropriate matching conditions at the internal vertices and boundary conditions at the external vertices. Following earlier work of Pivovarchik [14], we consider an inverse eigenvalue problem for a graph consisting of three equal length edges meeting at a single point, where the spectral data is the Dirichlet eigenvalues of the graph together with the Dirichlet spectra of the three individual edges. We derive, discuss and demonstrate a constructive solution method, obtain an alternative uniqueness proof, and discuss several kinds of generalizations.
\end{abstract}

Mathematics Subject Classification (2010). 34B24, 34A50, 34A55, 34L05, 81Q10.

Keywords. Inverse Sturm-Liouville problem, quantum graph.

\section{Introduction}

Consider the simple star graph consisting of 3 edges meeting at a common vertex, each edge of length 1 , with a real valued potential $q_{i} \in L^{\infty}(0,1)$ defined on the $i$ 'th edge, with edge parameterized by $[0,1]$ in such a way that $x=1$ corresponds to the common vertex where the edges meet. The direct eigenvalue problem consists in finding $\lambda \in \mathbb{C}$ and $\psi(x)=\left\{\psi_{1}(x), \psi_{2}(x), \psi_{3}(x)\right\}$ on $[0,1]$, with at least one

\footnotetext{
${ }^{1}$ Research supported by the National Science Foundation under grant DMS-1319052.

2 This work was carried out in part while both authors were visiting the Isaac Newton Institute for Mathematical Sciences, Cambridge UK.
} 
component non-zero, such that

$$
\begin{gathered}
\psi_{i}^{\prime \prime}+\left(\lambda-q_{i}(x)\right) \psi_{i}=0, \quad 0<x<1, i=1,2,3, \\
\psi_{1}(0)=\psi_{2}(0)=\psi_{3}(0)=0, \\
\psi_{1}(1)=\psi_{2}(1)=\psi_{3}(1), \quad \psi_{1}^{\prime}(1)+\psi_{2}^{\prime}(1)+\psi_{3}^{\prime}(1)=0 .
\end{gathered}
$$

The matching conditions (1.1) at the interior node are often referred to as the Kirchhoff conditions, by analogy with the condition for electric circuits that the sum of currents at each node must be zero. Denote the spectrum of this problem by $\left\{\lambda_{j}\right\}_{j=1}^{\infty}$, the natural frequencies of vibration for the graph - we will recall some of its properties below.

We also let $\left\{\mu_{i, j}\right\}_{j=1}^{\infty}, i=1,2,3$ denote the Dirichlet spectrum for the $i$ 'th edge. The following problem was studied by Pivovarchik in [14]:

Inverse spectral problem. Determine $q_{1}, q_{2}, q_{3}$ from the four spectra $\left\{\lambda_{j}\right\}$, $\left\{\mu_{i, j}\right\}, i=1,2,3, j=1,2, \ldots$

In particular existence and uniqueness results were proved under some restrictions, see below. The purpose of this note is to present a numerical solution method for Pivovarchik's problem, which is an adaptation of the method introduced in [18]. As a consequence of this we will be able to provide an alternative short and transparent proof of uniqueness.

The general topic of inverse Sturm-Liouville problems for graphs has attracted the attention of many researchers in recent years, see for example [1],[2], [5], [20], and references in these papers. See also [3] for a general introduction to the spectral theory of quantum graphs. As is well known even for the classical case of the inverse Sturm-Liouville problem on an interval, one spectrum is insufficient information to reconstruct the potential, aside from results that depend on having very specific values for the spectra such as those of Ambarzumian type (see e.g. Section 3.4 of [6]) or when the spectra has both real and complex parts due to non-self adjoint boundary conditions (see e.g. [4] or [17]). Thus we must expect to need to provide additional information besides the graph spectrum in order to recover the individual edge potentials. In these other recent works the additional information has consisted of suitably defined norming constants for the graph ([2]), further graph spectra obtained by changing the boundary condition at one or more of the boundary nodes ([20]) an appropriately defined Titchmarsh-Weyl function for the graph ([1] and [20]), or Dirichlet to Neumann map ([1] and [5]). Each of the above is in some sense an analogue of a known kind of spectral data for the case of a single interval. 
Pivovarchik has proposed and studied a graph inverse spectral problem of a somewhat different character, for a star graph consisting of $N$ edges of equal length meeting at one common vertex, where the Kirchhoff matching conditions are imposed, and using as data the $N+1$ sequences $\left\{\lambda_{j}\right\}_{j=1}^{\infty}$, the spectrum for the graph, and $\left\{\mu_{i, j}\right\}_{j=1}^{\infty}, i=1, \ldots N$, the Dirichlet spectra for each of the individual edges. See [16] for $N=2$, [14] for $N=3$, which is the inverse spectral problem stated above, and [15] for the general case. Although Pivovarchik's method is in a sense constructive, it relies on representations of entire functions with prescribed zero sets, and so does not seem well suited to actual computation. See also [7] for the $N=2$ case. We will focus on the $N=3$ case only, but there is no difficulty in adapting the method to any $N$, and also to the case of unequal edge lengths.

This problem may be viewed as a special case of a general class of inverse spectral problems for a metric graph, in which the given data consists of the natural frequencies of vibration of the entire graph, together with the natural frequencies for a certain number of its subgraphs. Cases of other possible matching conditions at the interior vertices, and other homogeneous boundary conditions at the boundary vertices, could also be considered.

\section{Direct and inverse problem}

Let us denote by $\phi_{i}(x, \lambda)$ the solution of

$$
\phi^{\prime \prime}+\left(\lambda-q_{i}(x)\right) \phi=0, \quad 0<x<1, \quad \phi(0)=0, \quad \phi^{\prime}(0)=1 .
$$

The roots of $\phi_{i}(1, \lambda)$ are the Dirichlet eigenvalues $\left\{\mu_{i, j}\right\}_{j=1}^{\infty}$ corresponding to $q_{i}$, and satisfy ${ }^{1}$

$$
\mu_{i, j}=(j \pi)^{2}+B_{i}+\ell^{2}, \quad j=1,2, \ldots,
$$

where $B_{i}=\int_{0}^{1} q_{i}(s) d s$.

In order that $\lambda$ be a graph eigenvalue, we must have

$$
\left\{\psi_{1}, \psi_{2}, \psi_{3}\right\}=\left\{\alpha_{1} \phi_{1}(\cdot, \lambda), \alpha_{2} \phi_{2}(\cdot, \lambda), \alpha_{3} \phi_{3}(\cdot, \lambda)\right\},
$$

with $\alpha_{1}, \alpha_{2}, \alpha_{3}$ not all zero.

${ }^{1}$ Here and below the $\ell^{2}$ symbol has the obvious meaning that

$$
\sum_{j=1}^{\infty}\left(\mu_{i, j}-(j \pi)^{2}-B_{i}\right)^{2}<\infty .
$$


From the matching conditions (1.1) we now obtain the three equations

$$
\begin{aligned}
\alpha_{1} \phi_{1}(1, \lambda)-\alpha_{2} \phi_{2}(1, \lambda) & =0 \\
\alpha_{1} \phi_{1}(1, \lambda)-\alpha_{3} \phi_{3}(1, \lambda) & =0 \\
\alpha_{1} \phi_{1}^{\prime}(1, \lambda)+\alpha_{2} \phi_{2}^{\prime}(1, \lambda)+\alpha_{3} \phi_{3}^{\prime}(1, \lambda) & =0
\end{aligned}
$$

and the zero determinant condition for a nontrivial solution to exist is then seen to be equivalent to

$$
\Phi(\lambda):=\left.\frac{d}{d x}\left(\phi_{1}(x, \lambda) \phi_{2}(x, \lambda) \phi_{3}(x, \lambda)\right)\right|_{x=1}=0 .
$$

We will always refer to $\Phi$ as the characteristic function for the graph eigenvalue problem.

Note that if $q_{i}(x) \equiv 0$ for $i=1,2,3$ then this equation becomes

$$
\frac{\sin ^{2}(\sqrt{\lambda}) \cos \sqrt{\lambda}}{\lambda}=0
$$

so that for each integer $j \geq 1, \lambda=(j \pi)^{2}$ is a double root, while $\left(\left(j-\frac{1}{2}\right) \pi\right)^{2}$ is a simple root. Under small perturbations we expect the double root generically to split into 2 simple roots, and this is indeed the case under some restrictions given below.

The graph spectrum is studied in detail in [14], where in particular (Lemma 1.17) it is shown that the sequence of graph eigenvalues may be regarded as the union of three subsequences $\left\{\lambda_{j, k}\right\}_{j=1}^{\infty}, k=1,2,3$ for which the following asymptotic expressions are valid:

$$
\lambda_{j, k}=(j \pi)^{2}+C_{k}+\ell^{2}, \quad k=1,2, j=1,2, \ldots,
$$

and

$$
\lambda_{j, 3}=\left(\left(j-\frac{1}{2}\right) \pi\right)^{2}+C_{3}+\ell^{2}, \quad j=1,2, \ldots
$$

Here the constants $C_{k}$ are related to the mean values $B_{k}$ by

$$
C_{3}=\left(B_{1}+B_{2}+B_{3}\right) / 3
$$

and $C_{1}, C_{2}$ are the two roots of the quadratic

$$
3 r^{2}-\frac{2}{\pi}\left(B_{1}+B_{2}+B_{3}\right) r+\frac{1}{\pi^{2}}\left(B_{1} B_{2}+B_{1} B_{3}+B_{2} B_{3}\right)=0 .
$$


The discriminant of this quadratic is

$$
\begin{aligned}
B_{1}^{2} & +B_{2}^{2}+B_{3}^{2}-B_{1} B_{2}-B_{1} B_{3}-B_{2} B_{3} \\
& =\frac{1}{2}\left(\left(B_{1}-B_{2}\right)^{2}+\left(B_{2}-B_{3}\right)^{2}+\left(B_{3}-B_{1}\right)^{2}\right) ;
\end{aligned}
$$

thus $C_{1}$ and $C_{2}$ are real and distinct provided at least one pair of edge potentials have distinct mean values. With this assumption we would know, at least for sufficiently large index, that there can be no multiple eigenvalues.

It is shown in [14], Corollary 1.24, that if we arrange the union of the three edge eigenvalue sequences $\left\{\mu_{i, j}\right\}_{j=1}^{\infty}, i=1,2,3$ into a single monotone sequence $\left\{\theta_{j}\right\}$, then the $\lambda_{j}, \theta_{j}$ sequences interlace,

$$
\lambda_{j} \leq \theta_{j} \leq \lambda_{j+1} \quad j=1,2 \ldots
$$

The possibility of equality occurring here cannot be ruled out, will certainly happen in some cases of interest, and loss of uniqueness will result (see for example [9] for a related example for the star graph consisting of only two edges). The assumptions made in this paper will guarantee that the inequalities in (2.8) are strict, see Lemma 3.1 below. In the case when equality occurs, one may then attempt to describe the corresponding isospectral set in detail, or identify some additional data which may serve to restore uniqueness. We believe that the constructive approach given in this paper may provide some insight towards the resolution of these questions. See also, the end of section 5 below.

In [14] the inverse spectral problem of recovering the three potentials $q_{i}$ from the four sequences of eigenvalues $\mu_{i, j}, \lambda_{j}$ is studied, and existence and uniqueness results are obtained, see Theorem 2.1 of [14]. We will not give the complete statement of this theorem here as it is somewhat lengthy and technical, but the essence is that a unique solution exists provided that the spectral sequences satisfy a certain asymptotic behavior which is a bit more restrictive than the necessary conditions given in (2.2), (2.5), and (2.6), (more or less equivalent to the requirement that $\left.q_{i} \in H^{1}(0,1)\right)$ and that $(2.8)$ with strict inequalities holds.

\section{Description of computational method for the inverse problem}

We now present the main outline of an algorithm for reconstructing the three edge potentials $q_{i}$ from the four eigenvalue sequences $\left\{\lambda_{j}\right\}_{j=1}^{\infty}$, and $\left\{\mu_{i, j}\right\}_{j=1}^{\infty}$, $i=1,2,3$, under the assumption that there is no overlap of the spectra. Numerical details will be given in section 5. As mentioned above, a uniqueness and 
existence result is proved for this inverse spectral problem in [14], under somewhat more restrictive conditions on the spectral data than have been mentioned so far. The only explicit assumption we make on the spectral data is that there is no overlap of the three edge spectra.

Lemma 3.1. If there is no overlap of the three edge spectra then an edge eigenvalue cannot be a graph eigenvalue. In particular (2.8) holds with strict inequalities.

Proof. By (2.3) we have

$$
\Phi\left(\mu_{1, j}\right)=\phi_{1}^{\prime}\left(1, \mu_{1, j}\right) \phi_{2}\left(1, \mu_{1, j}\right) \phi_{3}\left(1, \mu_{1, j}\right) .
$$

Neither of $\phi_{2}\left(1, \mu_{1, j}\right), \phi_{3}\left(1, \mu_{1, j}\right)$ can be zero by our assumption, and $\phi_{1}^{\prime}\left(1, \mu_{1, j}\right)$ cannot be zero either, since otherwise we'd have $\phi_{1}\left(1, \mu_{1, j}\right)=\phi_{1}^{\prime}\left(1, \mu_{1, j}\right)=0$. Thus $\mu_{1, j}$ is not a graph eigenvalue, and similarly for $\mu_{2, j}, \mu_{3, j}$.

Next, as is well known ([6], [8], [10], and [13]) we can represent the solution $\phi_{i}$ of (2.1) as

$$
\phi_{i}(x, \lambda)=\frac{\sin (\sqrt{\lambda} x)}{\sqrt{\lambda}}+\int_{0}^{x} K_{i}(x, t) \frac{\sin (\sqrt{\lambda} t)}{\sqrt{\lambda}} d t,
$$

for $i=1,2,3$. Here $K_{i}$ is the usual Gelfand-Levitan kernel corresponding to $q_{i}$, and which is known to also be a solution of the Goursat problem

$$
\begin{array}{cl}
K_{i t t}-K_{i x x}+q_{i}(x) K_{i}=0, & 0<t<x<1, \\
K_{i}(x, 0)=0, \quad K_{i}(x, x)=\frac{1}{2} \int_{0}^{x} q_{i}(s) d t, & 0<x<1 .
\end{array}
$$

This is classical ([8] and [13]) if $q_{i}$ is continuous. For the case $q_{i} \in L^{2}$ see [10], Theorem 4.18. It is known ([18], Theorem 1, and [10], Section 4.7) that the potential $q_{i} \in L^{\infty}(0,1)$ is uniquely determined by Cauchy data for $K$ for $x=1$, that is, by $\left\{K_{i}(1, t), K_{i x}(1, t)\right\}$ for $0 \leq t \leq 1$. Effective computational methods are available which will be recalled in the next section.

To determine the Cauchy data from the given spectral data, we begin by proceeding as in [18] to evaluate (3.1) at $\lambda=\mu_{i, j}$ and $x=1$ to get

$$
\int_{0}^{1} K_{i}(1, t) \sin \left(\sqrt{\mu_{i, j}} t\right) d t=-\sin \left(\sqrt{\mu_{i, j}}\right) .
$$

The three functions $K_{i}(1, t)$ are uniquely determined on $[0,1]$ by these inner products (see e.g. Lemma 1 of [18] or Theorem 4.20 of [10]), and it follows in particular that $\phi_{i}(1, \lambda)$ is uniquely determined for any $\lambda$. 
We now want to obtain the Neumann data $K_{i x}(1, t)$ for $i=1,2,3$. Rewrite the main equation (2.3) for the graph eigenvalues as

$$
\frac{\phi_{1}^{\prime}(1, \lambda)}{\phi_{1}(1, \lambda)}+\frac{\phi_{2}^{\prime}(1, \lambda)}{\phi_{2}(1, \lambda)}+\frac{\phi_{3}^{\prime}(1, \lambda)}{\phi_{3}(1, \lambda)}=0
$$

For $\lambda=\lambda_{j}$ no division by zero can occur here by Lemma 3.1.

A system of equations for the functions $K_{i x}(1, t)$ results by substituting from equation (3.1),

$$
\sum_{i=1}^{3} \frac{1}{\phi_{i}\left(1, \lambda_{j}\right)} \int_{0}^{1} K_{i x}(1, t) \sin \left(\sqrt{\lambda_{j}} t\right) d t=\Gamma_{j},
$$

where

$$
\Gamma_{j}=-\sum_{i=1}^{3} \frac{\sqrt{\lambda_{j}} \cos \sqrt{\lambda_{j}}+K_{i}(1,1) \sin \sqrt{\lambda_{j}}}{\phi_{i}\left(1, \lambda_{j}\right)} .
$$

Recall that $\phi_{i}(1, \lambda), K_{i}(1,1)$ are already known, thus $\Gamma_{j}$ may be evaluated directly from the given spectral data. Discussion of the unique solvability of (3.6) is given in the next section. Now given the three sets of Cauchy data $\left\{K_{i}(1, t), K_{i x}(1, t)\right\}$ it follows, as above, that the three edge potentials $q_{1}, q_{2}, q_{3}$ are uniquely determined, and may be obtained by known computational techniques.

\section{Uniqueness of the recovery of $K_{i x}(1, t)$}

We now explain why there is at most one solution of the system (3.6), from which it follows that uniqueness holds in the inverse spectral problem.

Multiply the homogeneous version of this system by $\lambda \prod_{i=1}^{3} \phi_{i}(1, \lambda)$ to get

$$
Z\left(\sqrt{\lambda_{j}}\right)=0, \quad j=1,2, \ldots,
$$

where

$$
\begin{gathered}
Z(\rho)=\zeta_{1}(\rho) \zeta_{2}(\rho) \hat{K}_{3}(\rho)+\zeta_{1}(\rho) \hat{K}_{2}(\rho) \zeta_{3}(\rho)+\hat{K}_{1}(\rho) \zeta_{2}(\rho) \zeta_{3}(\rho), \\
\zeta_{i}(\rho)=\sin \rho+\int_{0}^{1} K_{i}(1, t) \sin \rho t d t
\end{gathered}
$$

and

$$
\hat{K}_{i}(\rho)=\int_{0}^{1} K_{i x}(1, t) \sin \rho t d t .
$$

Note also that $Z$ is odd, and has a triple root at $\rho=0$. 
The function $Z$ is entire in $\rho$ and satisfies

$$
|Z(\rho)| \leq C e^{3|\rho|},
$$

so by the Paley-Wiener theorem there exists $z \in L^{2}(-3,3)$ such that

$$
Z(\rho)=\int_{-3}^{3} z(t) e^{-i \rho t} d t
$$

With an obvious rescaling, $\tilde{z}(t)=z\left(\frac{3}{\pi} t\right)$, we can write instead

$$
\tilde{Z}(\rho)=Z\left(\frac{\pi}{3} \rho\right)=\int_{-\pi}^{\pi} \tilde{z}(t) e^{-i \rho t} d t,
$$

and

$$
\tilde{Z}\left(\rho_{j}\right)=\int_{-\pi}^{\pi} \tilde{z}(t) e^{-i \rho_{j} t} d t=0, \quad j=1,2, \ldots,
$$

where

$$
\rho_{j}= \pm \frac{3}{\pi} \sqrt{\lambda_{j}}
$$

along with the triple root at $\rho=0$.

We now want to conclude that $\widetilde{Z}$, and hence $Z$, is identically zero, by appealing to a theorem of Levinson about completeness of sets of exponentials $\left\{e^{i \rho_{k} t}\right\}$. From this it will then follow that $K_{i x}(1, t)=0$ for $i=1,2,3$.

Theorem 4.1 (Theorem 3, p. 99 of [19] or Theorem III, p. 6 of [12]). Let $\left\{\rho_{k}\right\}$ be a sequence of complex numbers, $n(t)$ denote the number of $\rho_{k}$ satisfying $\left|\rho_{k}\right| \leq t$ and $N(r)=\int_{1}^{r} \frac{n(t)}{t} d t$ for $r>1$. Then it is sufficient for completeness of $\left\{e^{i \rho_{k} t}\right\}$ in $L^{p}(-\pi, \pi)$, that

$$
\limsup _{r \rightarrow \infty}\left(N(r)-2 r+\frac{1}{p} \log r\right)>-\infty .
$$

Multiple roots are allowed here, see p. 94 of [19].

First consider the case in which the actual $\rho_{j}$ values are replaced by their asymptotic limits, i.e. we use (2.5),(2.6) omitting the decaying $\ell^{2}$ error term. Keeping in mind the triple root of $\widetilde{Z}$ at $\rho=0$, this amounts to the property that the counting function $n(t)$ is

$$
n(t)= \begin{cases}6 k+3, & 3 k<t<3(k+1 / 2), \\ 6 k+5, & 3(k+1 / 2)<t<3(k+1),\end{cases}
$$

for $k=1,2, \ldots$ It follows that for a large integer $R$

$$
N(3 R+3)=\sum_{k=1}^{R}(6 k+3) \log \left(\frac{k+1 / 2}{k}\right)+(6 k+5) \log \left(\frac{k+1}{k+1 / 2}\right)+O(1) .
$$


Using

$$
\log (1+x)=x-\frac{x^{2}}{2}+O\left(x^{3}\right)
$$

we get

$$
(6 k+3) \log \left(\frac{k+1 / 2}{k}\right)=3+\frac{3}{4 k}+O\left(\frac{1}{k^{2}}\right)
$$

and

$$
(6 k+5) \log \left(\frac{k+1}{k+1 / 2}\right)=3+\frac{1}{4 k}+O\left(\frac{1}{k^{2}}\right) .
$$

Therefore

$$
N(3 R+3)=6 R+\log R+O(1) .
$$

Combining the above for $R^{\prime}=3 R+3$ yields

$$
\limsup _{R^{\prime} \rightarrow \infty} N\left(R^{\prime}\right)-2 R^{\prime}+\frac{1}{p} \log R^{\prime}>-\infty,
$$

as is needed for completeness, for any $p>1$.

If now we use the actual (rescaled) sequence $\rho_{j}$ instead of its asymptotic form, then because of the large $j$ behavior given by (2.5) and (2.6), it is not hard to see that the worst case is is when $\rho_{j}$ exceeds $j$ or $\left(j-\frac{1}{2}\right)$ by $O(1 / j)$. This in turn amounts to an $O\left(\frac{1}{j^{2}}\right)$ change in $N(r)$, and summing on $j$ we obtain at worst $O(1)$ total error, so that (4.1) is still valid.

We summarize our conclusions as

Theorem 4.2. Three potentials $q_{1}, q_{2}, q_{3} \in L^{\infty}(0,1)$ are uniquely determined by the spectral data $\lambda_{j}, \mu_{i, j}, i=1,2,3, j=1,2, \ldots$ provided that there is no overlap of the edge spectra $\mu_{i, j}$.

\section{Numerical examples}

We now provide details and discussion of some of the issues involved in the numerical implementation of the procedure discussed in Section 3 and illustrate with a numerical example. To recapitulate, the three main steps are

(1) obtain $K_{i}(1, t)$ for $i=1,2,3$ using (3.4);

(2) obtain $K_{i x}(1, t)$ for $i=1,2,3$ using (3.6);

(3) obtain the three edge potentials $q_{i}$ using one of the methods from [18]. 
We will actually replace $K_{i}(1, t)$ by $K_{i t}(1, t)$ in step 1 since it is more convenient in step 3 , and since $K_{i}(1,0)=0$, knowledge of $K_{i}(1, t)$ and $K_{i t}(1, t)$ are equivalent.

We assume we have available a finite number of spectral data $\lambda_{j}, j=1, \ldots M$, $\mu_{i, j}, j=1, \ldots N, i=1,2,3$ where $M=3 N$. As a preliminary step we do the customary estimation of the values of

$$
K_{i}(1,1)=\frac{1}{2} \int_{0}^{1} q(s) d s=\lim _{j \rightarrow \infty} \frac{1}{2}\left(\mu_{i, j}-(j \pi)^{2}\right) .
$$

The simplest estimate would be to take the 'last value', i.e.

$$
K_{i}(1,1) \approx \frac{1}{2}\left(\mu_{i, N}-(N \pi)^{2}\right)
$$

where $N$ is the index of the largest available eigenvalue. Provided $q$ is somewhat smooth we expect that the edge eigenvalue residues will follow a well defined pattern, thus extrapolation methods, such as the classical Aitken $\Delta^{2}$ acceleration method, could be used to advantage. As always, care must be exercised as such extrapolation methods for worst case scenarios can lead to poorer estimates.

For each fixed $i$, using a representation

$$
K_{i}(1, t)=K_{i}(1,1) t+\sum_{k=1}^{N} a_{i k} \sin k \pi t
$$

we get

$$
K_{i t}(1, t)=K_{i}(1,1)+\sum_{k=1}^{N} b_{i k} \cos k \pi t
$$

where the coefficients $b_{i k}$ are obtained, taking into account (3.4), from

$$
\sum_{k=1}^{N} M_{i j k} b_{i k}=-\sqrt{\mu_{i, j}} \sin \sqrt{\mu_{i, j}}+K_{i}(1,1)\left(\cos \sqrt{\mu_{i, j}}-\operatorname{sinc} \sqrt{\mu_{i, j}}\right)
$$

and $^{2}$

$$
M_{i j k}=\int_{0}^{1} \cos (k \pi t) \cos \left(\sqrt{\mu_{i, j}} t\right) d t=\frac{\sqrt{\mu_{i, j}}}{k \pi+\sqrt{\mu_{i, j}}} \operatorname{sinc}\left(\sqrt{\mu_{i, j}}-k \pi\right) .
$$

For potentials of any reasonable size the matrix $M_{i}=\left[M_{i j k}\right]$ is close to one half of the identity, hence very well conditioned.

\footnotetext{
${ }^{2}$ Here we are using the 'mathematics' version of the $\operatorname{sinc}$ function, $\operatorname{sinc}(z)=\sin z / z$.
} 
We also obtain immediately a corresponding expression for the edge characteristic function $\phi_{i}(1, \lambda)$ by substitution into (3.1) and some obvious manipulations, namely

$$
\begin{aligned}
\phi_{i}(1, \lambda)= & \operatorname{sinc} \sqrt{\lambda}+\frac{K_{i}(1,1)}{\lambda}(\cos \sqrt{\lambda}+\operatorname{sinc} \sqrt{\lambda}) \\
& +\frac{1}{\lambda} \sum_{k=1}^{N} b_{i k} \int_{0}^{1} \cos k \pi t \cos \sqrt{\lambda} t d t .
\end{aligned}
$$

We thus may evaluate the needed constants $\Gamma_{j}$ in(3.7) for $j=1, \ldots M$, using the previously obtained values of $b_{i k}$.

Finally we obtain an approximate solution of the system (3.6) by inserting a representation

$$
K_{i x}(1, t)=\sum_{k=1}^{N} c_{i k} \sin \left(k-\frac{1}{2}\right) \pi t,
$$

and solving the resulting $M \times M$ linear system for $\left\{c_{i k}\right\}_{k=1}^{N}, i=1,2,3$.

With approximations to $\left\{K_{i t}(1, t), K_{i x}(1, t)\right\}, 0<t<1$ in hand, we may now employ either of the methods given in [18] to obtain $q_{i}$ by solving the overdetermined Cauchy problem defined by (3.2) and (3.3) and the given $K_{i t}(1, t), K_{i x}(1, t)$.

To produce a numerical example, we have generated spectral data with high accuracy using the program MATSLISE [11] for the individual edge eigenvalues $\mu_{i, j}$ with the edge coefficients chosen to be ${ }^{3}$

$$
\begin{aligned}
& q_{1}(x)=3|x-.4|, \\
& q_{2}(x)=3 e^{x} \sin 4 \pi x, \\
& q_{3}(x)=\chi_{[0, .4]}(x)+3 \chi_{[.4, .8]}(x),
\end{aligned}
$$

which have varying degrees of smoothness, distinct mean values, and none of which can be considered as small.

Evaluation of $\Phi$, which of course amounts to evaluation of $\phi_{i}(1, \lambda)$, and $\phi_{i}^{\prime}(1, \lambda)$ for $i=1,2,3$, is done by solving the initial value problem (2.1) by means of of an ODE solver with a stringent error tolerance and potentials $q_{i}$ provided as explicit functions. For eigenvalues less than $\lambda=500$ we used the standard routine RKF, and for larger values we switched to a lower order solver adapted for stiff equations. To locate the graph eigenvalues $\lambda_{j}$, a simple linear bisection algorithm was used to find the zeros of the characteristic function $\Phi$ to a specified tolerance. Recall by the interlacing property mentioned in section 2 , that if $\theta_{0}, \ldots \theta_{M}$ denote

\footnotetext{
${ }^{3}$ Here $\chi_{E}$ denotes the usual indicator function of the set $E$
} 
the numbers $\left\{0, \mu_{i, j}: i=1,2,3 j=1, \ldots N\right\}$ in increasing order then we must have $\lambda_{j} \in\left(\theta_{j-1}, \theta_{j}\right), j=1, \ldots M$. We therefore use $\theta_{j-1}, \theta_{j}$ as the endpoints of an initial bracketing interval for $\lambda_{j}$, to ensure that no eigenvalue is omitted. Due to the asymptotic behavior (2.2), (2.5) (or the reasons mentioned after (2.4)), we may expect that the some of the spectral data are very closely spaced, specifically near any $(k \pi)^{2}$ we will find all three $\mu_{i, k}$ and 2 terms from the graph eigenvalue sequence $\lambda_{j}$. Table 1 displays the first few (square roots of) edge and graph eigenvalues for the example (5.2) to illustrate this point.

Table 1. Spectral data for example (5.2).

\begin{tabular}{||l|l|l|l||}
\hline$\sqrt{\mu_{1, j}}$ & $\sqrt{\mu_{2, j}}$ & $\sqrt{\mu_{3, j}}$ & $\sqrt{\lambda_{j}}$ \\
\hline \multirow{3}{*}{3.2205} & & & \\
& & & 1.6518 \\
& 3.1451 & & 3.1835 \\
6.3429 & & 3.4780 & 3.3897 \\
& & & 4.6925 \\
& 6.2490 & & 6.2935 \\
& & 6.4086 & 6.3802 \\
& & & 7.9285 \\
& 9.3899 & & 9.4205 \\
& & 9.5097 & 9.4898 \\
\hline
\end{tabular}

In Figures 1 and 2 we show the coefficient reconstructions obtained using $N=10$, and $N=5$ respectively. The relative $L^{2}(0,1)$ errors in recovery of the three coefficients, for $N=10$ are approximately $3 \%, 6 \%$ and $15 \%$ for $q_{1}, q_{2}, q_{3}$ respectively, or $9 \%$ in terms of the vector $\left\langle q_{1}, q_{2}, q_{3}\right\rangle$. For $N=5$ the corresponding numbers are $10 \%, 14 \%, 22 \%$ and $16 \%$. One can improve the accuracy obviously by using more pieces of spectral data, but even without this there would be a significant improvement if we were able to use more accurate values of $K_{i}(1,1)$.

We close this section by making a few remarks about the case when two edge spectra overlap, say the $m$ 'th Dirichlet spectra of edges 1 and 2, $\mu_{1, m}=\mu_{2, m}$. The computation of $K_{i}(1, t)$ for each edge goes through as before but the matrix $\mathcal{K}$ involved in (3.6) will now have one row which must be deleted, since a division by zero occurs, so there will be a one dimensional null space for the remaining system. There are several ways to proceed to restore uniqueness by adding a free parameter to the system and we mention only one possibility for which we make no claim of optimality as the entire issue of degeneracy of the spectra is delicate and certainly worthy of further study. 

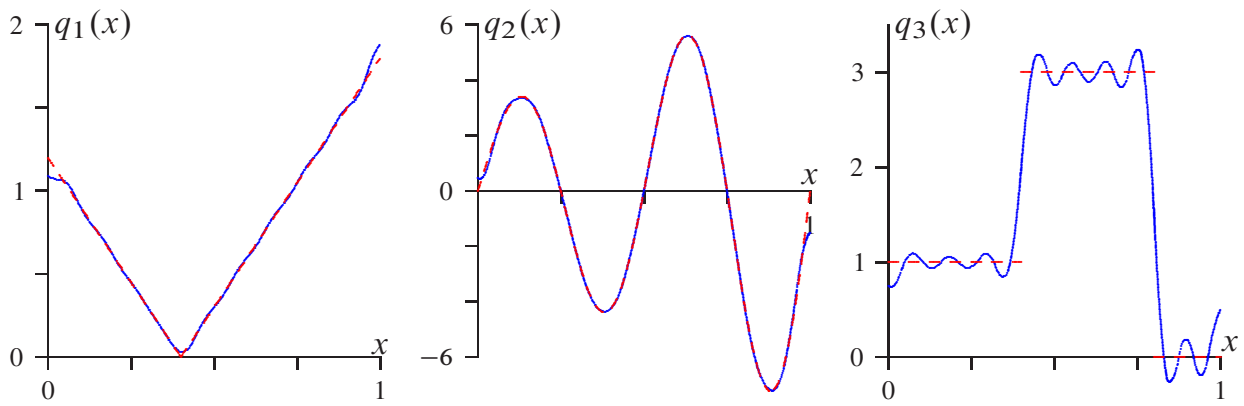

Figure 1. Numerical reconstructions of $q_{1}, q_{2}, q_{3}$ with $N=10$.
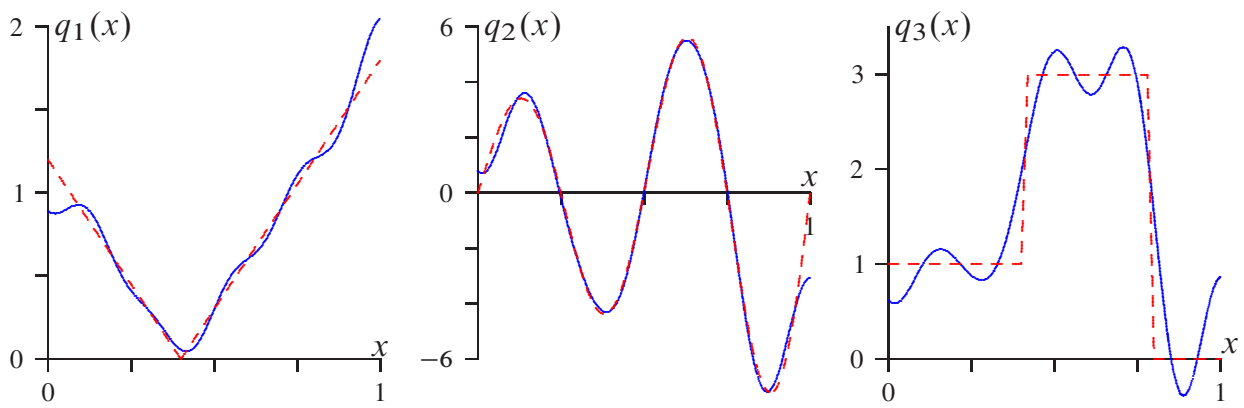

Figure 2. Numerical reconstructions of $q_{1}, q_{2}, q_{3}$ with $N=5$.

One can split the double root by replacing it by $\mu_{1, m}+\delta$ and $\mu_{2, m}-\delta$ for some value $\delta$. The question is how to select $\delta$. We clearly have to also avoid $\mu_{3, m}$ and our suggestion is to select the smallest $\delta$ that does not increase the condition number of the matrix formed from $\mathcal{K}$ by deleting the $m$ 'th row (which is now nonsingular). An alternative is to take $\delta$ such that the separation becomes equal to the minimum separation amongst other edge spectra and one should expect these two approaches are related.

\section{More general star graphs}

There are two obvious generalizations which we can make without any new difficulties, at least from the computational point of view. One is to allow for unequal edge lengths $L_{1}, L_{2}, L_{3}$, in which case the characteristic function determining the graph eigenvalues would have to be written as

$$
\begin{aligned}
& \Phi(\lambda):=\phi_{1}\left(L_{1}, \lambda\right) \phi_{2}\left(L_{2}, \lambda\right) \phi_{3}^{\prime}\left(L_{3}, \lambda\right)+\phi_{1}\left(L_{1}, \lambda\right) \phi_{2}^{\prime}\left(L_{2}, \lambda\right) \phi_{3}\left(L_{3}, \lambda\right) \\
& \quad+\phi_{1}^{\prime}\left(L_{1}, \lambda\right) \phi_{2}\left(L_{2}, \lambda\right) \phi_{3}\left(L_{3}, \lambda\right),
\end{aligned}
$$


while (3.6) and (3.7) become

$$
\sum_{i=1}^{3} \frac{1}{\phi_{i}\left(L_{i}, \lambda_{j}\right)} \int_{0}^{1} K_{i x}\left(L_{i}, t\right) \sin \left(\sqrt{\lambda_{j}} t\right) d t=\Gamma_{j},
$$

and

$$
\Gamma_{j}=-\sum_{i=1}^{3} \frac{\sqrt{\lambda_{j}} \cos \sqrt{\lambda_{j}}+K_{i}\left(L_{i}, L_{i}\right) \sin \sqrt{\lambda_{j}}}{\phi_{i}\left(L_{i}, \lambda_{j}\right)} .
$$

It is important to note in this case that it is no longer appropriate to use the same number of eigenvalues for each edge, rather we fix an upper bound $\Lambda_{\max }$ and use only the edge and graph eigenvalues satisfying $\mu_{i, j}, \lambda_{i} \leq \Lambda_{\max }$. One therefore ends up using $N_{i} \approx\left(L_{i} / \pi\right) \sqrt{\Lambda_{\max }}$ eigenvalues from the $i$ 'th edge. The graph and edge eigenvalues still interlace ${ }^{4}$ and in this way we may guarantee that equal total numbers of edge and graph eigenvalues are used, with the number of edge eigenvalues being weighted by the length of the edge. Correspondingly we use $N_{i}$ terms in the representation (5.1) of $K_{i x}(1, t)$. Note that the edge lengths $L_{i}$ themselves are easily estimated from the $\mu_{i, j}$ 's by means of the asymptotic behavior

$$
\mu_{i, j}=\left(\frac{j \pi}{L_{i}}\right)^{2}+\frac{1}{L_{i}} \int_{0}^{L_{i}} q_{i}(s) d s+\ell^{2} .
$$

For example, we have carried out the indicated procedure with the coefficients defined as in (5.2) with edge lengths chosen to be $L_{1}, L_{2}, L_{3}=1,1.2, .75$ and $\Lambda_{\max }=1000$, so that respectively 10,7 and 12 eigenvalues are used from each edge sequence and 17 graph eigenvalues. The relative errors in $L^{2}\left(0, L_{i}\right)$ are 9,10 and $12 \%$ respectively, or about $10 \%$ in terms of $\left\langle q_{1}, q_{2}, q_{3}\right\rangle$ This is comparable or slightly larger than in the case of equal edge lengths, mainly due to a slightly poorer estimate of $K_{i}\left(L_{i}, L_{i}\right)$. If instead we chose the edge lengths to be $(1, .95,1.05)$ then the overall relative error is slightly smaller than in the equal edge length case.

One may also allow for a star graph consisting of $n>3$ edges meeting at a common vertex, which is the case studied in [15]. Here the graph eigenvalues are the roots of the characteristic function defined as in (2.3) except with $n$ terms in the product, and we need only modify (3.6), (3.7) by replacing $\sum_{i=1}^{3}$ by $\sum_{i=1}^{n}$.

\section{Factors influencing the condition number}

It may be expected that the discretized version of the system (3.6) is rather illconditioned due to small divisors, i.e. the fact that one of the terms $\phi_{i}\left(1, \lambda_{j}\right) \approx 0$

\footnotetext{
${ }^{4}$ This is an empirical observation only, and not directly relevant to our discussion.
} 
whenever $\sqrt{\lambda_{j}}$ is in close proximity to one of the values $\sqrt{\mu_{i, j}}$. This is essentially guaranteed to hold in the equal edge length case, due to the fact that

$$
\sqrt{\mu_{i, j}} \approx j \pi+\frac{B_{i}}{2 j \pi}, \quad B_{i}=\bar{q}_{i}=\int_{0}^{1} q_{i}(s) d s,
$$

and because the graph eigenvalues always lie between adjacent edge eigenvalues. In the above example (5.2), with $M=3 N=15$ we find $\kappa_{2} \approx 6 \times 10^{2}$, while if $M=3 N=30$ it would be about $2 \times 10^{4}$. By contrast, for the classical single interval inverse spectral problem, the analogous condition number is around 1 and largely independent of $M$. If we multiply each $q_{i}$ by 2 , so the minimum difference of the mean values $\bar{q}_{i}$ is doubled, the condition number is reduced by about $1 / 2$ and the same happens if the potentials are doubled again.

For the same reasons, the situation would be much worse if two of, or all three, edge potentials had the same mean value, since then the edge eigenvalues are much more closely clustered together, forcing the same to be true of the graph eigenvalues as well. Thus we are likely to see even smaller divisors in (3.6). If, for example, in (5.2) we replace $q_{1}(x)$ by 5.12|x-1/3|, so that $q_{1}, q_{3}$ have the same mean value, then the condition numbers rise dramatically, $3 \times 10^{4}$ for $M=15$ and $6 \times 10^{5}$ for $M=30$.

By the same reasoning, we expect a smaller condition number when the edge lengths are unequal, since then there will be much less tendency for edge and graph eigenvalues to be close to each other. In the case of (5.2), for example, with with edge lengths chosen to be $L_{1}, L_{2}, L_{3}=1,1.05, .95$, the corresponding condition number is merely 18 for $M=30$.

A natural question is how the condition number of the matrix occurring in (3.6) changes with $M$ and also with the spacing between the values of $B_{i}$ in (2.2). Our system (3.6) and (3.7) is based on equation (3.5) which was obtained from the definition of $\Phi$ in (2.3) by dividing through by the product $\phi_{1}(1, \lambda) \phi_{2}(1, \lambda) \phi_{3}(1, \lambda)$. While (3.6) provides a simple representation, this may not be optimal for numerical computations as both the matrix entry $1 / \phi_{i}\left(1, \lambda_{j}\right)$ and the corresponding right hand side term $\Gamma_{j}$ has now a division by a quantity that can become arbitrary small for large enough spectral index. Indeed, we know that two in every group of three graph spectra must accumulate at $n \pi$ for integral $n$ and since there is an edge spectra in between, at least one of the terms in each row of the equation as well as the right hand side must in fact grow approximately at least linearly with $n$ as the differences of the edge spectra is $O(1 / n)$ from the asymptotic formula (2.2).

This suggest that we rescale the equations by dividing each row by its largest term in magnitude - or for each $j$ by multiplying by the value $\phi_{i}\left(1, \lambda_{j}\right)$ for some $i$. In a sense this is just partially reversing the transformation of (2.3) into (3.5) and 
is a preconditioning of the system (3.6), (3.7) by means of an initial pivoting strategy. This also has the advantage of ensuring our new right hand side $\widetilde{\Gamma}_{j}$ remains uniformly bounded for all indices $j$ - just as in the case of a single interval - and allows us to make comparisons between condition numbers for various values of $M$ and the spacing of the values $B_{i}$.

To test the conditioning of the problem we chose $q_{i}=2 /\left(x+a_{i}\right)^{2}$, functions where the form of the eigenfunctions are known explicitly, ${ }^{5}$ in order to reduce computational error in the entries of the matrix.

We then computed the condition number as a function of $N$ from $N=5, \ldots 30$. To test the dependence on the separation of the values $B_{i}=\int_{0}^{1} q_{i}(s) d s$ we chose $a_{2}=0.5$ and adjusted $a_{1}$ and $a_{2}$ to make the $B_{1}-B_{2}=B_{2}-B_{3}=\delta$ for some given $\delta$. The results are shown in Figure 3 for $B_{i}$ differences of $0.25,0.1$ and 0.05 . Here $\kappa(N)$ is computed for the rescaled equations with a uniformly bounded right hand side rather than (3.7) itself. For a fixed value of $\delta$, the linear dependence of the condition number in terms of $N$ is clearly shown. We should remark that for other potentials a similar linear relationship appears to hold, although not always to the same precision as shown here. The most important feature though is the strong dependence on $\delta$ which plays at least as large a role as the number of spectral values.

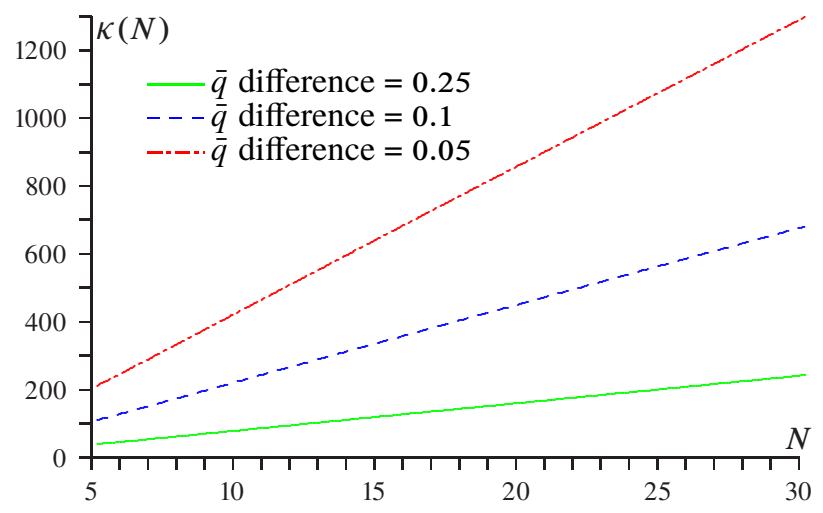

Figure 3. Behavior of condition number for the recovery of $K_{i x}(1, t)$.

Finally, we must also expect the degree of ill-conditioning to increase when the number of edges increases, but the rate of increase seems to be modest. For example if we try a case with $n=5$, using edge potentials for which the relative spacing of the mean values is comparable to the case (5.2) with $q_{1}, q_{2}, q_{3}$ only, then the condition number for the analogue of (3.6) is typically within a factor of 2 of the corresponding condition number for $n=3$.

${ }^{5}$ Any eigenfunction has the form $\frac{\sin (k x)}{k}+\frac{\sin (k x)-k x \cos (k x)}{k^{3} a_{i}\left(x+a_{i}\right)}$, where $k^{2}$ is an eigenvalue. 


\section{References}

[1] S. Avdonin and P. Kurasov, Inverse problems for quantum trees. Inverse Probl. Imaging 2 (2008), no. 1, 1-21. MR 2375320 Zbl 1148.35356

[2] M. I. Belishev, Boundary spectral inverse problem on a class of graphs (trees) by the BC method. Inverse Problems 20 (2004), no. 3, 647-672. MR 2067494 Zbl 1086.35122

[3] G. Berkolaiko and P. Kuchment, Introduction to quantum graphs. Mathematical Surveys and Monographs, 186. American Mathematical Society, Providence, R.I., 2013. MR 3013208 Zbl 06132651

[4] B. M. Brown, I. Knowles, and R. Weikard, On the inverse resonance problem. J. London Math. Soc. (2) 68 (2003), no. 2, 383-401. MR 1994689 Zbl 1050.34004

[5] B. M. Brown and R. Weikard, A Borg-Levinson theorem for trees. Proc. R. Soc. Lond. Ser. A Math. Phys. Eng. Sci. 461 (2005), no. 2062, 3231-3243. MR 2172226 Zbl 05213594

[6] K. Chadan, D. Colton, L. Päivärinta, and W. Rundell, An introduction to inverse scattering and inverse spectral problems. With a foreword by M. Cheney. SIAM Monographs on Mathematical Modeling and Computation. Society for Industrial and Applied Mathematics (SIAM), Philadelphia, PA, 1997. MR 1445771 Zbl 0870.35121

[7] M. C. Drignei, Constructibility of an $L_{\mathbb{R}}^{2}(0, a)$ solution to an inverse Sturm-Liouville problem using three Dirichlet spectra. Inverse Problems 26 (2010), no. 2, Article id. 025003, 29 pp. MR $2575360 \mathrm{Zbl} 1211.34010$

[8] I. M. Gel'fand and B. M. Levitan, On the determination of a differential equation from its spectral function. Izvestiya Akad. Nauk SSSR. Ser. Mat. 15 (1951). 309-360. In Russian. English transl., Amer. Math. Soc. Transl. (2) 1 (1955), 253-304. MR 0045281 MR 0073805 (transl.) Zbl 0044.09301 Zbl 0066.33603 (transl.)

[9] F. Gesztesy and B. Simon, On the determination of a potential from three spectra. In V. Buslaev, M. Solomyak, and D. Yafaev (eds.), Differential operators and spectral theory. M. Sh. Birman's 70 ${ }^{\text {th }}$ anniversary collection. American Mathematical Society Translations, Series 2, 189. Advances in the Mathematical Sciences, 41. American Mathematical Society, Providence, R.I., 1999, 85-92. MR 1730505 MR 1730498 (collection) Zbl 0922.34008 Zbl 0911.00011 (collection)

[10] A. Kirsch, An introduction to the mathematical theory of inverse problems. Applied Mathematical Sciences, 120. Springer, Berlin etc., 1996. MR 1479408

Zbl 0865.35004

[11] V. Ledoux, M. Van Daele, and G. Vanden Berghe, MATSLISE: A MATLAB package for the numerical solution of Sturm-Liouville and Schrödinger equations. ACM Trans. Math. Software 31 (2005), no. 4, 532-554. MR 2272344 Zbl 1136.65327

[12] N. Levinson, Gap and density theorems. American Mathematical Society Colloquium Publications, 26, American Mathematical Society, New York, 1940. MR 0003208 Zbl 0145.08003 
[13] B. M. Levitan, Inverse Sturm-Liouville problems. Nauka, Moscow, 1984. In Russian. English transl. by O. Efimov. VNU Science Press, Utrecht, 1987. MR 0771843 MR 0933088 (transl.) Zbl 0575.34001 Zbl 0749.34001 (transl.)

[14] V. Pivovarchik, Inverse problem for the Sturm-Liouville equation on a simple graph. SIAM J. Math. Anal. 32 (2000), no. 4, 801-819. MR 1814739 Zbl 0993.34025

[15] V. Pivovarchik, Inverse problem for the Sturm-Liouville equation on a star-shaped graph. Math. Nachr. 280 (2007), no. 13-14, 1595-1619. MR 2354982 Zbl 1135.34304

[16] V. N. Pivovarchik, An inverse Sturm-Liouville problem by three spectra. Integral Equations Operator Theory 34 (1999), no. 2, 234-243. MR 1694710 Zbl 0948.34014

[17] W. Rundell and P. Sacks, Numerical technique for the inverse resonance problem. J. Comput. Appl. Math. 170 (2004), no. 2, 337-347. MR 2075015 Zbl 1055.65086

[18] W. Rundell and P. E. Sacks, Reconstruction techniques for classical inverse Sturm-Liouville problems. Math. Comp. 58 (1992), no. 197, 161-183. MR 1106979 Zbl 0745.34015

[19] R. M. Young, An introduction to nonharmonic Fourier series. Revised edition. Academic Press, Inc., San Diego, CA, 2001. MR 1836633 Zbl 0981.42001

[20] V. Yurko, Inverse spectral problems for Sturm-Liouville operators on graphs. Inverse Problems 21 (2005), no. 3, 1075-1086. MR 2146822 Zbl 1089.34009

Received September 29, 2013; revised November 19, 2013

William Rundell, Department of Mathematics, Texas A\&M University,

College Station, TX 77843, U.S.A.

e-mail: rundell@math.tamu.edu

Paul Sacks, Department of Mathematics, Iowa State University, Ames, IA 50011, U.S.A.

e-mail: psacks@iastate.edu 